\title{
Epidemiological dynamics of bacteriocin competition and antibiotic resistance
}

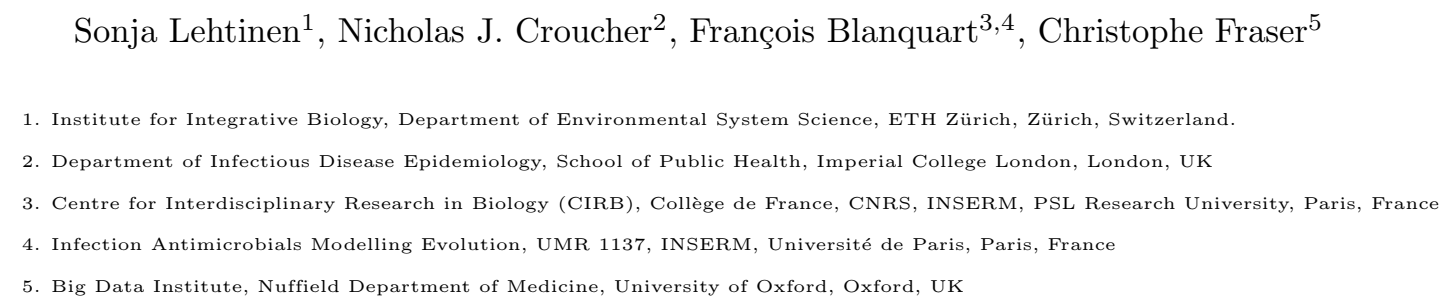

Bacteriocins, toxic peptides involved in bacterial fratricide, are extremely diverse. Understanding the mechanisms that maintain this diversity is an important aim in bacterial ecology. Previous work on bacteriocin diversity has focused on dynamics, particularly 'rock-paper-scissors' dynamics, at the within-host scale. Yet, in species such as Streptococcus pneumoniae, with relatively short periods of colonisation and limited within-host diversity, processes at the epidemiological scale also shape eco-evolutionary dynamics. Here, we investigate bacteriocin dynamics in epidemiological models. We find that in these models, bacteriocin diversity arises more readily than in within-host models, and with more possible combinations of coexisting bacteriocin profiles. We also investigate a potential link between bacteriocin diversity and diversity at antibiotic resistance loci. Previous work has proposed that bacterial duration of carriage modulates the fitness of antibiotic resistance. We predict bacteriocins modulate duration of carriage, making them a plausible candidate for involvement in resistance dynamics.

\section{Introduction}

Bacteriocins are toxic peptides that allow bacteria to eliminate competitors. Almost all bacteria have bacteriocin systems and these systems are thought to be a significant component of competition within (and possibly between [1]) bacterial species [2]. A mechanistic understanding of the role of bacteriocins in shaping competition is therefore important for characterising the eco-evolutionary dynamics of bacteria.

At a high level of abstraction, bacteriocin systems can be thought of as consisting of three components: a system to produce and secrete toxins, a system to achieve immunity against these toxins, and a regulatory system to control toxin and immunity production (which may involve signaling components allowing quorum-sensing and kin recognition). Even with these three components, bacteriocin systems are highly diverse. A single species may have a number of distinct bacteriocin systems, with considerable diversity within each of these: coexistence of strains with different combinations of toxin, immunity and regulatory genes is pervasive [3].

Previous work on the eco-evolutionary dynamics of bacteriocin systems has sought to explain how this diversity arises and persists. Two-strain models of toxin-producing ('producer') and toxin-susceptible ('non-producer') strains do not predict coexistence: depending on the effectiveness of the toxins and the cost associated with their production, either the producer or the non-producer strategy out-competes the other $[4,5]$. Coexistence can be achieved by inclusion of a third ('immune') strain which does not produce toxins but does have immunity against them (or, alternatively, produces less effective and less costly toxins [5]). If both toxin and immunity incur a fitness cost, the total cost to the producer strain (which has both toxins and immunity), 
is greater than the total cost to the immune strain (which only pays the cost for immunity). As a consequence, in head-to-head competition, the non-producer out-competes the immune strain; the immune strain out-competes the producer; and the producer out-competes the non-producer (if the cost of toxins and immunity are not too high). This type of non-transitive competitive structure is referred to as 'rock-paper-scissors' dynamics [6].

In the context of bacteriocin dynamics, it is worth making a distinction between two different rock-paper-scissors structures. In the most general formulation of rock-paper-scissors competition, strain interactions are modelled in terms of the outcome of head-to-head competition (Figure $1 \mathrm{~A}$ ). The mechanism leading to this outcome (e.g. differences in growth rate due to fitness costs, the toxin-induced killing of non-producer cells) is not explicitly modelled. Strain identities are therefore interchangeable: each strain wins against one of the other strains and loses against the other, but there are no further ecological properties that distinguish one strain from another. Models building on this structure have been studied in a number of ecological contexts [6], including bacteriocin dynamics [7]. Such models predict oscillatory dynamics of the three strains in well-mixed environments and stable coexistence in spatially structured environments $[6,7]$.

On the other hand, bacteriocin-specific formulations of rock-paper-scissors competition explicitly model the killing of the non-producers by the producers, as well as the lower growth rate of immune and producer strains arising from fitness costs (Figure $1 \mathrm{~B}$ ). The non-transitive structure in head-to-head competition is retained, but the strains have different ecological properties. For example, the toxin-induced death of non-producer cells leads to freeing up of resources (e.g. nutrients or space), which can then be used by any of the strains. As a result, the immune strain gains the same benefit from the production of toxins as the producer strain, without paying the cost (a 'cheater' strategy). These bacteriocin-specific models, like general rock-paper-scissors models, predict stable coexistence of the three strains in spatially structured environments, but dominance of a single strain in well-mixed systems [5]. This prediction has been verified in an experimental model of producer, non-producer and immune strain interactions in Escherichia coli $[8]$.

These bacteriocin-specific models include differences in growth rate and toxin-induced death, but exclude processes relating to the transmission of bacteria between hosts (i.e. establishment and clearance of bacterial carriage). In other words, the insights above are derived from models at the within-host, not epidemiological, scale. Yet, in some species, such as Streptococcus pneumoniae, epidemiological dynamics likely play a significant role in the evolution of bacteriocin systems, for two reasons. Firstly, within-host models assume all three species are co-colonising the same host. However, multiple colonisation is not necessarily the norm: for example, reported rates of co-colonisation with multiple pneumococcal strains are around $25 \%$ to $50 \%$ depending on setting $[9,10,11]$. Secondly, when the duration of colonisation is relatively short (e.g. of the order of months in S. pneumoniae), factors affecting transmission will play a large role in shaping allele frequencies in the overall bacterial population.

The distinction between within-host and epidemiological scales matters: the nature of strain interactions depends on the scale at which these interactions are considered (Figure $1 \mathrm{C}$ ). Firstly, the assumption that the killing of non-producer cells leads to resources being freed-up only applies at the within-host scale. At the epidemiological scale, when a producer strain invades a host carrying a non-producer strain, the host is not freed-up, but colonised by the producer. The immune strain gains no 'cheater' benefit. Secondly, recent modelling suggests that when the benefits of immunity are shared, rather than specific to the immune strain (e.g. immunity involves secretion of toxin-degrading compounds), coexistence can also arise in well-mixed environments [12]. However, such effect can only occur at the within-host scale. Finally, considered at the epidemiological scale, the fitness cost of toxins and immunity may impact the relative fitness of strains when they compete within the same host, but also a strain's growth rate when it is the sole coloniser and therefore epidemiological parameters like transmission and clearance rate.

For these reasons, epidemiological models are necessary for understanding bacteriocin dynamics and diversity in S. pneumoniae and species with similar properties. A further motivation for developing such models is that bacteriocins potentially play a role in determining bacterial duration of carriage. We have previously suggested that duration of carriage affects selection pressure for antibiotic resistance in species which are carried asymptomatically most of the time, such as S. pneumococcus [13]. Strains with a longer duration of carriage gain a greater fitness 


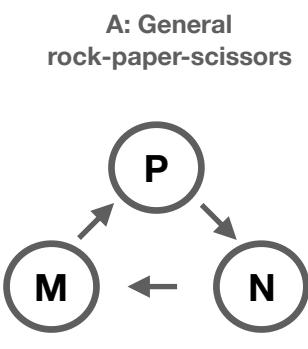

$$
\begin{aligned}
& \text { B: Bacteriocin-specific } \\
& \text { Within-host }
\end{aligned}
$$

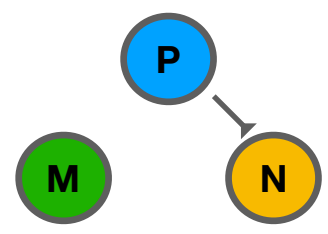

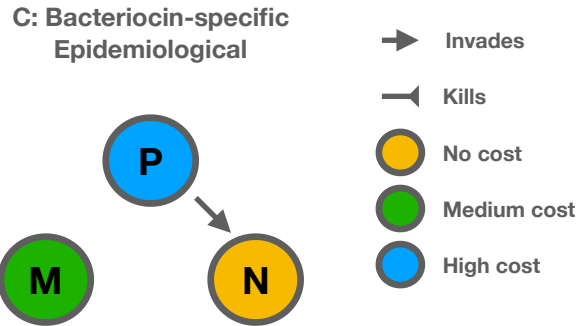

Figure 1: Strain interactions arising in different models of bacteriocin dynamics. $P$ represents the producer strain (both toxins and immunity); $M$ the immune strain (immunity only) and $N$ the non-producer (no toxins, no immunity). A: the general formulation of rock-paper-scissors competition, where strain interactions are modelled in terms of the outcome of head-to-head competition. The costs of toxins and immunity, as well as the killing action of the toxins, are modelled implicitly in the outcome of head-to-head competition. At the epidemiological scale, assuming fitness differences between strains are only apparent when the strains are competing for the same host gives rise to this model structure ('head-to-head competition' model, Equations 1). B: Bacteriocin-specific within-host model [5, 8], where the action of toxins, as well as the cost of toxin-production and immunity are modelled explicitly. The killing of the non-producer strain by the producer strain leads to resources being freed up. C: Bacteriocinspecific epidemiological model. The killing of the non-producer strain by the producer strain leads to the host becoming colonised with the producers strain. Assuming the fitness cost of toxin-production and immunity affects epidemiological parameters leads to the 'epidemiological costs' model structure (Equation 2).

benefit from resistance, leading to an association between long duration of carriage and antibiotic resistance (including multi-drug resistance [14]). As a result, balancing selection maintaining diversity at a locus affecting duration of carriage could also maintain diversity at a resistance locus [13], providing a potential explanation to the long-standing puzzle of why antibiotic resistance has not yet reached fixation [15]. However, this explanation is not complete, because the genetic determinants of duration of carriage have not been fully identified [16]. A more complete understanding of resistance dynamics therefore requires identifying loci that contribute to variation in duration of carriage. The role of toxin production in killing competing bacteria and the role of immunity in preventing this killing makes bacteriocin loci a promising candidate.

This paper is organised in two parts: the first part develops an epidemiological model of bacteriocin dynamics in S. pneumoniae (or a species with similar ecology). We explore the circumstances in which this model allows coexistence of strains with different bacteriocin profiles. In the second part, we investigate differences in duration of carriage between bacteriocin profiles and their impact on resistance dynamics.

\section{Part I: Bacteriocin dynamics}

\section{Epidemiological modelling of bacteriocin dynamics}

\section{Model structures}

We begin by considering a bacteriocin system with two components: a toxin gene and an immunity gene. There are therefore three possible strains: a producer strain $(P)$, with both toxin and immunity; an immune strain $(M)$, with immunity but no toxin; and a non-producer strain $(N)$, with neither toxin nor immunity (i.e. the entire bacteriocin locus is absent). Strains with the toxin but no immunity are assumed to be lethal to themselves and therefore not included. Bacteriocin production has been shown to inhibit competing strains in vitro and in murine models of colonisation (see, for example [17]). However, how this effect translates to the epidemiological level is not entirely clear (see, for example [18]). In light of this uncertainty, we model two distinct ways in which the bacteriocin production could influence epidemiological dynamics in a homogeneously mixing host population.

Head-to-head competition model: In this model, fitness differences between strains 
are apparent when strains are competing for the same host. A strain can invade an already colonised host if it has a fitness advantage over the resident strain. (For simplicity, we do not model co-infection: the invading strain out-competes the resident strain instantaneously; hosts are therefore only ever colonised with a single strain at a time). All strains colonise uncolonised individuals $X$ at rate $\beta$ and are cleared at rate $\mu$. Toxin production allows strain $P$ to outcompete strain $N$; strain $P$ is therefore able to invade hosts carrying the strain $N$ at rate $k_{P} \beta$. Strain $M$ out-competes strain $P$ because of the cost of toxin production; strain $M$ can therefore replace strain $P$ at rate $k_{M} \beta$. Strain $N$ out-competes strain $M$ because of the cost of immunity, and can therefore replace it in carriage at rate $k_{N} \beta$. The $k$ parameters reflect the probability of invasion relative to colonisation of an uncolonised host; for each $k, 0<k \leq 1$. These assumptions lead to the same model structure as the general rock-paper-scissors model of competition [6]. The dynamics of this model (Figure 1 A), which we refer to as the 'head-to-head competition,' are described by the following equations:

$$
\begin{aligned}
\frac{d I_{P}}{d t} & =\beta I_{P}\left(X+k_{P} I_{N}\right)-k_{M} \beta I_{M} I_{P}-\mu I_{P} \\
\frac{d I_{M}}{d t} & =\beta I_{M}\left(X+k_{M} I_{P}\right)-k_{N} \beta I_{N} I_{M}-\mu I_{M} \\
\frac{d I_{N}}{d t} & =\beta I_{N}\left(X+k_{N} I_{M}\right)-k_{P} \beta I_{P} I_{N}-\mu I_{N}
\end{aligned}
$$

Here, the $X$ and $I$ variables represent proportions of the total host populations: $X+I_{P}+$ $I_{M}+I_{N}=1$.

Epidemiological costs model: The second model differs from the first in two ways. Firstly, we assume invasion of an already colonised host requires toxin-induced killing of the resident strain. Thus, only the $P$ strain can invade an already colonised host: $k_{M}=k_{N}=0$. Secondly, instead of the cost of toxin and immunity affecting invasion of already colonised hosts, these costs affect the transmission and/or clearance rate. Producer strains incur fitness $\operatorname{costs} c_{P}^{\beta}$ and $c_{P}^{\mu}$ in transmission and clearance respectively, so that $\beta_{P}=\left(1-c_{P}^{\beta}\right) \beta$ and $\mu_{P}=\frac{\mu}{\left(1-c_{P}^{\mu}\right)}$. Similarly, the immune strain incurs a fitness cost such that $\beta_{M}=\left(1-c_{M}^{\beta}\right) \beta$ and $\mu_{M}=\frac{\mu}{\left(1-c_{M}^{\mu}\right)}$. The cost to the $P$ strain is higher than to the $M:\left(1-c_{P}^{\beta}\right)\left(1-c_{P}^{\mu}\right)<\left(1-c_{M}^{\beta}\right)\left(1-c_{M}^{\mu}\right)$. The dynamics of this model (Figure 1 C), which we refer to as the 'epidemiological costs' model are described by:

$$
\begin{array}{r}
\frac{d I_{P}}{d t}=\beta_{P} I_{P}\left(X+k_{P} I_{N}\right)-\mu_{P} I_{P} \\
\frac{d I_{M}}{d t}=\beta_{M} I_{M} X-\mu_{M} I_{M} \\
\frac{d I_{N}}{d t}=\beta I_{N} X-k_{P} \beta_{P} I_{P} I_{N}-\mu I_{N}
\end{array}
$$

There are two things to note about these model structures. Firstly, these two model structures represent two extreme cases of a more general model formulation. In this more general model formulation (see Supporting Information Section 1.3), the fitness cost of toxin-production and immunity can affect both the probability of successful invasion and the epidemiological parameters. For clarity, we begin by analysing these two extremes, and explore a hybrid model later.

Secondly, we have described bacteriocins as offensive (i.e. enabling invasion of already colonised hosts). It could also be that the role of bacteriocins is defensive (i.e. preventing invasion by another strain) or a combination of both. The distinction between offensive and defensive is only meaningful when the transmission rate differs between strains (see Supporting Information Section 1.3), and in practice, does not have an impact on the coexistence of bacteriocin strains (Supporting Figure 2).

\section{Approach to analysis of models}

To understand the circumstances under which strain diversity is maintained, i.e. whether there are stable equilibria with non-zero frequencies of more than one strain given the constraints 


Parameter
Transmission rate
Natural clearance rate
Successful invasion (producer)
Successful invasion (immune)
Successful invasion (non-producer)
Cost of toxin production (transmission)
Cost of toxin production (clearance)
Cost of immunity (transmission)
Cost of immunity (clearance)
Additional effect on transmission

$\begin{array}{lll}\text { Symbol } & \text { Constraints } & \text { Model(s) } \\ \beta & \beta>0 & \text { All } \\ \mu & \mu<\beta & \text { All } \\ k_{P} & 0<k_{P} \leq 1 & \text { All } \\ k_{M} & 0<k_{M} \leq 1 & \text { Head-to-head competition } \\ k_{N} & 0<k_{N} \leq 1 & \text { Head-to-head competition } \\ c_{P}^{\beta} & 0 \leq c_{P}^{\beta} \leq 1 & \text { Epidemiological costs } \\ c_{P}^{\mu} & 0 \leq c_{P}^{\mu} \leq 1 & \text { Epidemiological costs } \\ c_{M}^{\beta} & 0 \leq c_{M}^{\beta} \leq 1 & \text { Epidemiological costs } \\ c_{M}^{\mu} & 0 \leq c_{M}^{\mu} \leq 1 & \text { Epidemiological costs } \\ \rho & \rho>-1 & \text { Hybrid }\end{array}$

Table 1: Table of parameters, which model(s) they are relevant to and limits they are subject to. In addition to the limits in the table, we require that toxin production, immunity and resistance have an overall fitness cost (either in transmission or clearance): $\left(1-c^{\beta}\right)\left(1-c^{\mu}\right)<1$ for each trait.

on the parameters in Table 1, we performed linear stability analysis (details in Supporting Information Section 1). In general, when we present results for specific parameter values, we use $\mu=1$ and $\beta=2$ or $\beta=4$. In units of month $^{-1}$, these are reasonable values for $S$. pneumoniae. All analyses and simulations were performed using Wolfram Mathematica [19]; the code is available as a Supporting File.

\section{Bacteriocin diversity in the basic models}

\section{Both model structures predict balancing selection on bacteriocin locus}

Both the 'head-to-head competition' and 'epidemiological costs' models give rise to balancing selection on the bacteriocin locus, allowing coexistence of strains with different bacteriocin profiles. In line with previous results in models with the same structure [6], the 'head-to-head competition' model predicts coexistence of all three strains, with oscillatory dynamics around a stable equilibrium point (i.e. 'centre') (Figure 2 and Supporting Figure 1). These oscillations are not structurally stable: they may be sensitive to even minor changes in model structure (see below). Coexistence requires all three strains: equilibria involving just two of the strains are never stable (see Supporting Information Section 1.2).

The epidemiological costs model, on the other hand, predicts an asymptotically stable equilibrium, which, depending on parametrisation, includes just the non-producer strain (i.e. the locus is absent), the non-producer and producer strains, or all three strains (Figure 2 and Figure 4). Behaviour in this model therefore differs from models of within-host bacteriocin dynamics, in which the non-producer and producer strains without the immune strain is not observed $[5,8]$. This difference arises because, in the within-host model, the producer kills the non-producer, whereas in the epidemiological model, the producer replaces the non-producer strain. The fitness benefit of bacteriocin production is therefore directly dependent on the frequency of the non-producer strain, giving rise to negative frequency-dependent selection.

It is worth noting that the dynamics of the two and three strain equilibria are different. Rockpaper-scissors dynamics are known to give rise to 'survival of the weakest', where increasing the competitive advantage of a strain (e.g. a strain's $k$ in the head-to-head competition model) decreases its frequency [6]. This behaviour is also observed when the three strains are present in the epidemiological costs model, but not in the two strain (producer and non-producer) equilibrium (see Supporting Figure 7).

\section{Presence of epidemiological fitness differences leads to asymptotic stability}

The different dynamics predicted by the head-to-head competition and epidemiological costs models are interesting for two reasons. Firstly, in previous models of bacteriocin dynamics, asymptotic stability only arises in the presence of spatial structure $[6,7,5,8]$; we would therefore like to identify the factors giving rise to asymptotic stability in a well-mixed system. Secondly, the difference between these two behaviours impacts our understanding of how a bacterial population responds to perturbations. With asymptotic stability, the effect of perturbations is 

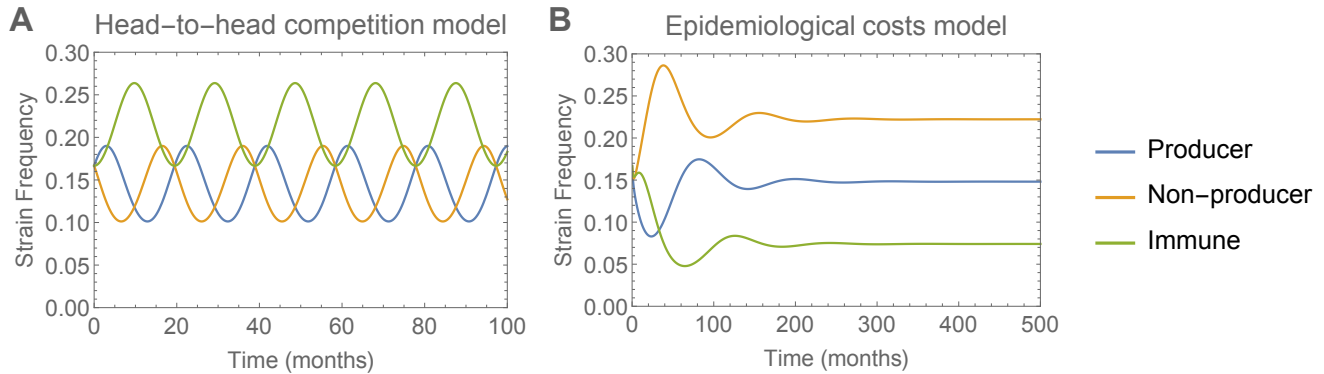

Figure 2: Examples of the dynamics of the head-to-head competition (A) and epidemiological costs (B) models. Parameters for these plots were: $\beta=2, \mu=1, k_{P}=0.5$ and, for plot A $k_{M}=0.75, k_{N}=0.5$ and, for plot $\mathrm{B} c_{P}^{\beta}=0.25, c_{M}^{\beta}=0.1$ and no costs associated with clearance.

temporary: pre-perturbation allele frequencies will eventually be recovered. With oscillation on the other hand, the oscillations will continue around the same centre, but the perturbation may permanently alter their amplitude. Public health interventions can be viewed as perturbations to the pathogen ecology; the difference between asymptotic stability and oscillation therefore has implications for predicting the response of pathogen populations to interventions such as vaccination $[20,21,22]$.

Oscillation arises when strains differ in which already colonised hosts they can invade, but not in their basic epidemiological parameters $(\beta$ and $\mu)$. We therefore explored the effect of introducing a difference in these epidemiological parameters into the head-to-head competition model (Equations 1). We introduce a parameter $\rho(\rho>-1)$ that scales the transmission rate of the producer strain. This gives the producer strain either an additional advantage $(\rho>0$, reflecting, for example, toxins also being effective against other nasopharyngeal species, leading to increased probability of colonisation) or an additional fitness cost $(\rho<0$ reflecting, for example, decreased growth rate leading a lower rate of transmission). The dynamics of this model (which we refer to as 'hybrid model') are described by:

$$
\begin{gathered}
\frac{d I_{P}}{d t}=(1+\rho) \beta I_{P}\left(X+k_{P} I_{N}\right)-k_{M} \beta I_{M} I_{P}-\mu I_{P} \\
\frac{d I_{M}}{d t}=\beta I_{M}\left(X+k_{M} I_{P}\right)-k_{N} \beta I_{N} I_{M}-\mu I_{M} \\
\frac{d I_{N}}{d t}=\beta I_{N}\left(X+k_{N} I_{M}\right)-k_{P}(1+\rho) \beta I_{P} I_{N}-\mu I_{N}
\end{gathered}
$$

Introduction of any difference in transmission rate $(\rho \neq 0)$ leads this model to behave like the original epidemiological cost model: equilibria are asymptotically stable and coexistence of two bacteriocin profiles is possible (Figure 3). Similar behaviour is seen when the additional cost or advantage of bacteriocin production affects the clearance, rather than transmission rate (see Supporting Figure 8). Thus, like spatial structure in previous models $[5,6,8]$, differences in the epidemiological parameters of strains act to stabilise coexistence.

\section{The effects of kin recognition (pherotype)}

\section{Extension of models to include pherotype}

Two of the best described pneumococcal bacteriocin systems, the blp [23, 24, 17, 25] and cib [26] loci, are regulated through a two-component regulatory system. Cells produce and release a signalling molecule, which, at high enough extra-cellular concentrations, is recognised by cells with a corresponding receptor, leading to expression of the bacteriocin locus. This allows both quorum-sensing (limiting expression of the locus to times when the producer density is high enough to produce sufficient levels of toxins for effective killing) and kin recognition (limiting activation of the locus to cells of the same 'pherotype' - i.e. with a receptor capable of detecting the specific signalling molecule). 


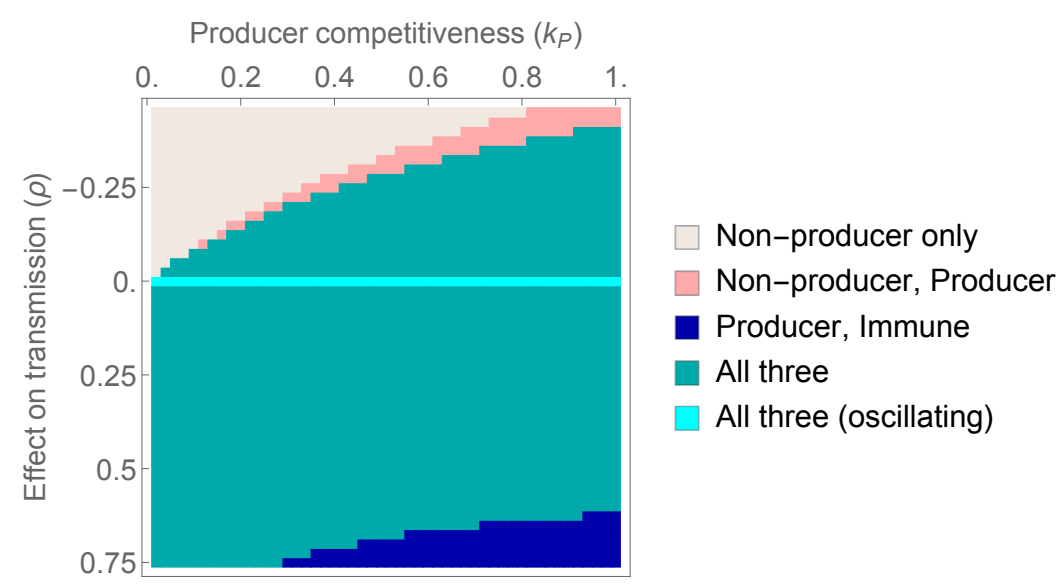

Figure 3: The effect of including fitness effect on transmission in the head-to-head competition model. The colours indicate which strains are present at equilibrium. Results are derived from a linear stability analysis and results shown are for asymptotically stable equilibria unless otherwise indicated. Model parameters were $\beta=2, \mu=1, k_{N}=0.5, k_{M}=0.5$.

We explore the effect of including a pheromone signalling system on the dynamics of the two basic models. The dynamics in both models remain similar to the original models (Equations 1 and 2), with the exception that immunity is not effective between pherotypes: the immune strain does not recognise the signal to turn on expression of immunity proteins when this signal arises from a producer of a different pherotype. Producer strains are therefore able to outcompete immune strains with a different pherotype. We assume producer strains of different pherotypes cannot invade each other, although this assumption does not impact model dynamics (Supporting Information Section 1.4).

For a system with two pherotypes $a$ and $b$, the dynamics of the head-to-head competition model are described by:

$$
\begin{aligned}
\frac{d I_{a, P}}{d t} & =\beta I_{a, P}\left(X+k_{P}\left(I_{a, N}+I_{b, N}+I_{b, M}\right)\right)-k_{M} \beta I_{a, M} I_{a, P}-\mu I_{a, P} \\
\frac{d I_{a, M}}{d t} & =\beta I_{a, M}\left(X+k_{M} I_{a, P}\right)-k_{N} \beta\left(I_{a, N}+I_{b, N}+I_{b, P}\right) I_{a, M}-\mu I_{a, M} \\
\frac{d I_{a, N}}{d t} & =\beta I_{a, N}\left(X+k_{N}\left(I_{a, M}+I_{b, M}\right)\right)-k_{P} \beta\left(I_{a, P}+I_{b, P}\right) I_{a, N}-\mu I_{a, N} \\
\frac{d I_{b, P}}{d t} & =\beta I_{b, P}\left(X+k_{P}\left(I_{a, N}+I_{b, N}+I_{a, M}\right)\right)-k_{M} \beta I_{b, M} I_{b, P}-\mu I_{b, P} \\
\frac{d I_{b, M}}{d t} & =\beta I_{b, M}\left(X+k_{M} I_{b, P}\right)-k_{N} \beta\left(I_{a, N}+I_{b, N}+I_{a, P}\right) I_{b, M}-\mu I_{b, M} \\
\frac{d I_{b, N}}{d t} & =\beta I_{b, N}\left(X+k_{N}\left(I_{a, M}+I_{b, M}\right)\right)-k_{P} \beta\left(I_{a, P}+I_{b, P}\right) I_{b, N}-\mu I_{b, N}
\end{aligned}
$$

And the dynamics of the epidemiological costs model are described by: 


$$
\begin{array}{r}
\frac{d I_{a, P}}{d t}=\beta_{P} I_{a, P}\left(X+k_{P}\left(I_{a, N}+I_{b, N}+I_{b, M}\right)\right)-\mu_{P} I_{a, P} \\
\frac{d I_{a, M}}{d t}=\beta_{M} I_{a, M} X-k_{P} \beta_{P} I_{b, P} I_{a, M}-\mu_{M} I_{a, M} \\
\frac{d I_{a, N}}{d t}=\beta I_{a, N} X-k_{P} \beta_{P}\left(I_{a, P}+I_{b, P}\right) I_{a, N}-\mu I_{a, N} \\
\frac{d I_{b, P}}{d t}=\beta_{P} I_{b, P}\left(X+k_{P}\left(I_{a, N}+I_{b, N}+I_{a, M}\right)\right)-\mu_{P} I_{b, P} \\
\frac{d I_{b, M}}{d t}=\beta_{M} I_{b, M} X-k_{P} \beta_{P} I_{a, P} I_{b, M}-\mu_{M} I_{b, M} \\
\frac{d I_{b, N}}{d t}=\beta I_{b, N} X-k_{P} \beta_{P}\left(I_{a, P}+I_{b, P}\right) I_{b, N}-\mu I_{b, N}
\end{array}
$$

\section{Inclusion of pherogroup allows coexistence without the non-producer strain}

For both the head-to-head competition and epidemiological costs models, the addition of pherotype increases the number of possible combinations in which strains can coexist, but decreases the parameter space in which coexistence of different bacteriocin profiles is observed (Figure 4). In the models without pherotype, production of bacteriocins is only beneficial if there are also strains susceptible to the bacteriocins (i.e. non-producer strains) - all stable equilibria therefore have a non-zero frequency of the non-producer strain. Inclusion of pherogroup expands the range of strains susceptible to the toxins, thus allowing the producer strain to exist without the non-producer strain.

In the head-to-head competition model with pherotype, producers exclude both other profiles when the competitiveness of the non-producer strain is greater than the competitiveness of the immune strain $\left(k_{P}>k_{M}\right)$. Similarly, in the epidemiological costs model, some parameter values which previously allowed coexistence of all three bacteriocin profiles now only feature either the producer and the immune strains or the producer strain only. In this model, the inclusion of pherotype introduces oscillation when both the immune and producer strains are present, although exploring the system's behaviour numerically suggests the amplitude of this oscillation is small.

\section{Part II: Potential role for bacteriocins in resistance dynam- ics}

As discussed in part I, the interaction of strains with different bacteriocin profiles gives rise to balancing selection maintaining diversity in these profiles. If different bacteriocin profiles are also associated with different durations of carriage, this balancing selection acting on the bacteriocin locus could also maintain coexistence of antibiotic sensitive and resistant strains. We therefore examine the extent of variation in duration of carriage between strains with different bacteriocin profiles. For simplicity, we only present results from the epidemiological costs model (without pherotype) in the main text. Results from the head-to-head competition model are qualitatively similar and included in Supporting Information (Section 2.1)

\section{Bacteriocin profiles differ in duration of carriage}

The mean duration of carriage of strain $i\left(D_{i}\right)$ is the inverse of its overall clearance rate, and therefore depends on the natural clearance rate $\mu$, any fitness costs affecting the clearance rate of strain $i$, and on the rate at which strain $i$ is replaced by another strain. In the epidemiological costs model, the duration of carriage of each strain are given by: 

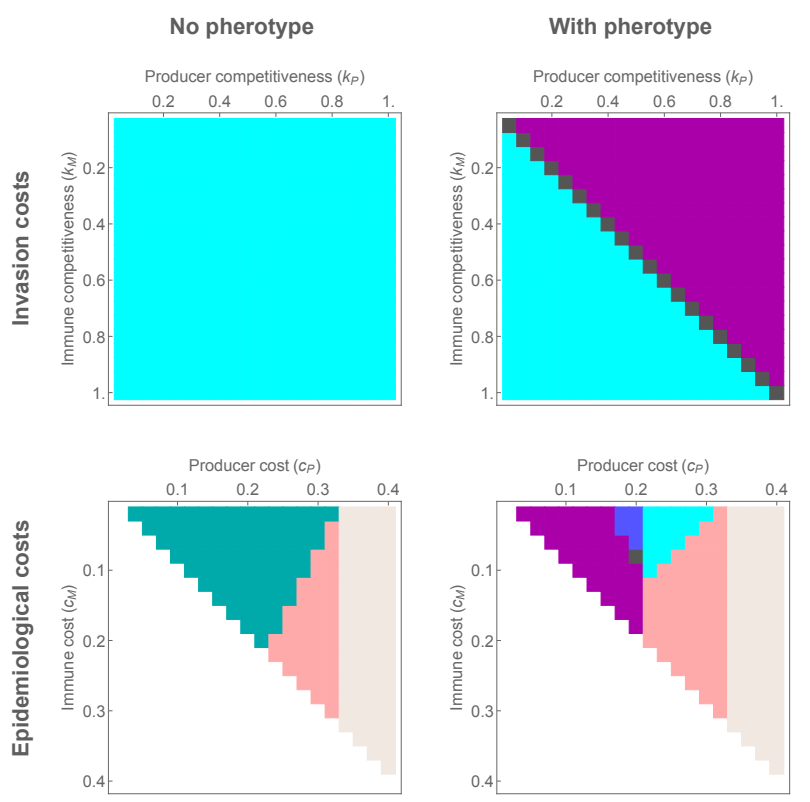

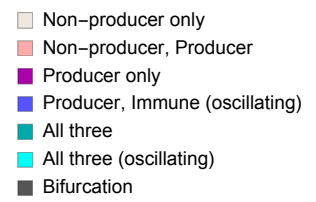

Bifurcation

Figure 4: The effect of kin recognition on the coexistence of different bacteriocin profiles in the head-to-head competition (top) and epidemiological costs (bottom) models. The colours indicate which strains are present at equilibrium, with (right) and without (left) inclusion of pherotype. The white space in the epidemiological costs models arises because we require $c_{P}>c_{M}$. Results are derived from a stability analysis and results shown are for asymptotically stable equilibria unless otherwise indicated. Model parameters were $\beta=2, \mu=1$, for both plots and $k_{N}=0.5$ for the head-to-head competition model and $k_{P}=0.5$ for the epidemiological cost model. In the epidemiological costs model, the costs of bacteriocin production and immunity were modelled to affect transmission only $\left(c_{P}^{\mu}=c_{I}^{\mu}=0\right)$. Results were qualitatively similar when these costs were modelled in clearance (Supporting Figure 9).

$$
\begin{array}{r}
D_{P}=\frac{\left(1-c_{P}^{\mu}\right)}{\mu} \\
D_{M}=\frac{\left(1-c_{M}^{\mu}\right)}{\mu} \\
D_{N}=\frac{1}{k_{P}\left(1-c_{P}^{\beta}\right) \beta I_{P}+\mu}
\end{array}
$$

In this model, the relative duration of carriage of different bacteriocin profiles depends primarily on how the cost of toxins and immunity is modelled (Figure 5). When these costs are entirely associated with transmission, differences in duration of carriage are entirely due to strain replacement. The non-producer strain, which is susceptible to invasion, therefore has a shorter duration of carriage than the other two strains. As the proportion of the cost associated with clearance rate increases, the duration of carriage of the immune and producer strains decreases. The duration of carriage of the non-producer strain is not greatly affected: although, according to Equation $6, D_{N}$ would increase with $c_{P}^{\beta}$ (and therefore should decrease with the proportion of costs associated with clearance rate), this effect is modulated by the frequency of the producer strain $\left(I_{P}\right)$. The result is that the duration of carriage of the non-producer strain is only marginally affected by the modelling of the costs of toxins and immunity. Therefore, when costs are primarily associated with clearance rate, the non-producer strain has a longer duration of carriage than the producer strain. Note that when the stable equilibrium includes the immune strain, this strain always has the longest duration of carriage. This is because it incurs a smaller fitness cost than the producer strain and, unlike the non-producer strain, is not susceptible to replacement.

The relatively modest variation in duration of carriage shown in Figure 5 is typical under 

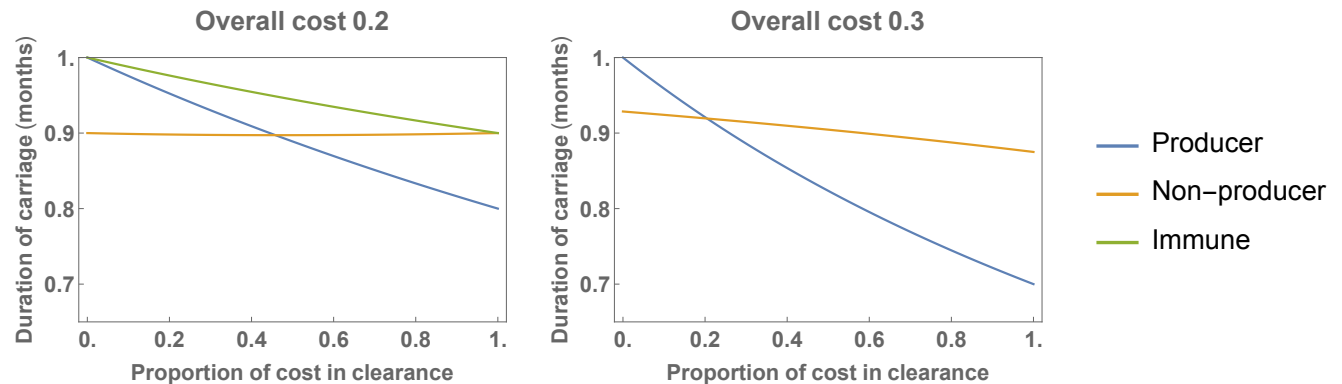

Figure 5: Variation in the duration of carriage (i.e. one over the overall clearance rate) of strains with different bacteriocin profiles. For each plot, the overall effect of fitness costs on the reproductive number of producer strain is kept constant (i.e. $\left(1-c_{P}^{\mu}\right)\left(1-c_{P}^{\beta}\right)$ is constant), but the proportion of the cost affecting clearance $\left(c_{P}^{\mu}\right)$ and transmission $\left(c_{P}^{\beta}\right)$ is varied. The overall cost refers to $1-\left(1-c_{P}^{\mu}\right)\left(1-c_{P}^{\beta}\right)$. The fitness costs for the immune strain are fixed at half those of the producer strain $c_{P}^{\mu}=2 c_{M}^{\mu}$ and $c_{P}^{\beta}=2 c_{M}^{\beta}$. Other parameters are $\beta=2, \mu=1, k_{P}=1 / 2$

most parameter ranges (Supporting Figure 10). However, more significant variation is observed under specific conditions: when the cost of toxins and immunity is very high and associated with clearance rate (Supporting Figure 10). Increasing the transmission rate $(\beta)$ or the invasiveness of the producer strain $\left(k_{P}\right)$ increases both the parameter space in which variation in duration of carriage is seen and the extent of this variation. This is because increasing $\beta$ and $k_{P}$ allows coexistence of producers and non-producers at higher fitness costs of toxin production (Supporting Figure 10).

\section{The effect of bacteriocins on antibiotic resistance dynamics}

The observed variation in the duration of carriage of different bacteriocin profiles suggests bacteriocins will indeed affect resistance dynamics. However, for this effect to be observed, the balancing selection on the bacteriocin locus must be strong enough to maintain this diversity in presence of antibiotics [13]. Furthermore, the results above do not give us information about the expected frequency of resistance. To investigate the effect of bacteriocins on antibiotic resistance dynamics in more detail, we therefore expand the epidemiological costs model to include competition between resistant and sensitive strains.

We model a species that is carried asymptomatically most of the time (e.g. S. pneumoniae) - the antibiotic exposure of hosts is therefore independent of whether they are colonised and therefore equal to average consumption rate of the antibiotic $(\tau)$. Interactions between the three bacteriocin profiles are the same as in the basic model (Equation 2). In addition, each profile can be either antibiotic sensitive $(S)$ or antibiotic resistant $(R)$, giving rise to six possible strains. Antibiotic sensitive strains are subject to an additional clearance rate $\tau$ (we assume immediate clearance in response to antibiotics). Resistance carries a fitness cost, which, for clarity, we assume affects transmission $\left(c_{R}^{\beta}\right)$ in the main text. The full model structure is given in the Supporting Information Section 2.2.

The results we present are robust to modelling the cost of resistance as affecting clearance rate, as long as this costs affects all components of clearance, i.e. increasing both natural clearance $\mu$ and, in the case of the non-producer strain, susceptibility to replacement by the producer strain. If this assumption does not hold, the difference in duration of carriage of the different bacteriocin profiles will not always impact resistance dynamics (see Supporting Information Section 2.2).

As expected, the between-strain variation in duration of carriage allows coexistence of antibiotic sensitivity and resistance, with antibiotic resistance associated with the bacteriocin profile(s) with longer duration of carriage (Figure 6). In line with the relatively modest variation in duration of carriage, the range of antibiotic consumption rates at which coexistence is observed is relatively narrow for most parametrisations. The frequency of resistance in the region of coexistence depends on the relative frequencies of the bacteriocin profiles. The intermediate resistance frequency the model gives rise to is therefore dependent on parameters that affect 

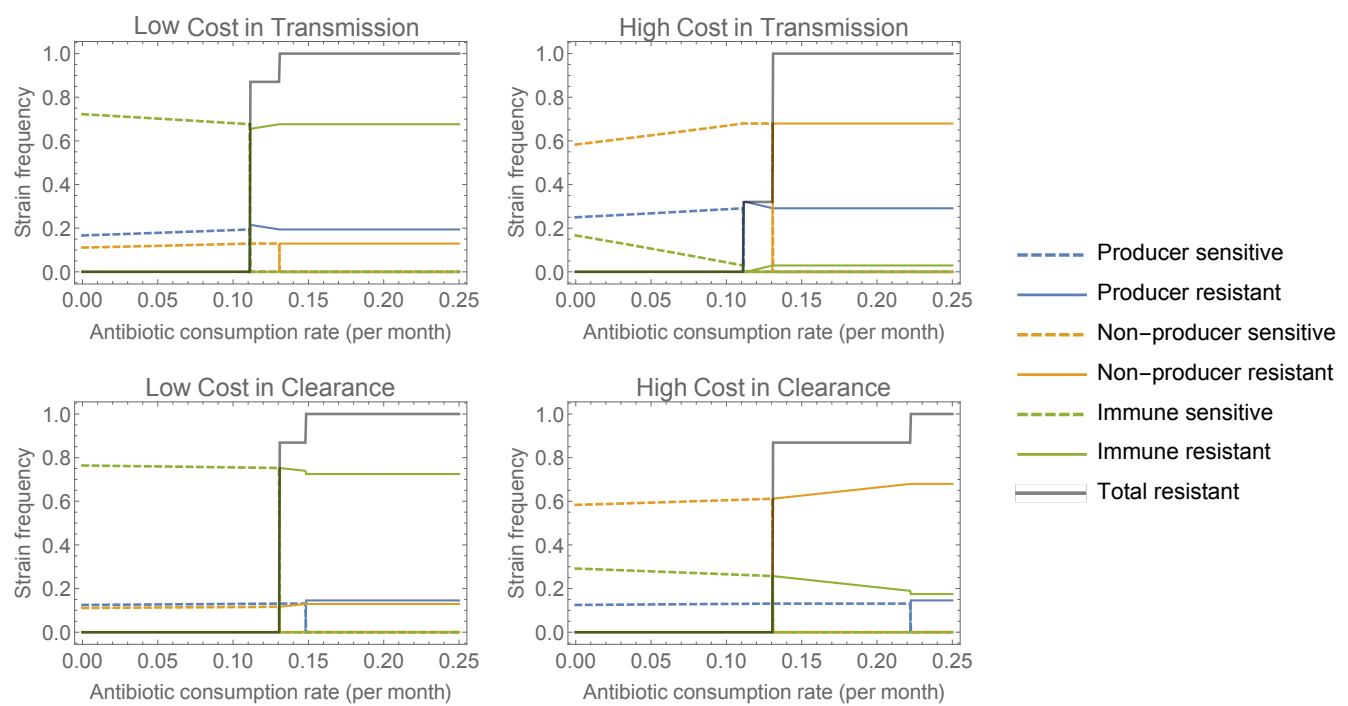

Figure 6: Strain frequencies (colours) and overall resistance frequency (black) as a function of antibiotic consumption rate $(\tau)$ for different parametrisation of costs for the producer strain. High cost is $c_{P}=0.5$ and low cost is $c_{P}=0.25$ and this cost is associated fully with either transmission or clearance. Other parameters are $\mu=1, k_{P}=0.5, c_{M}=0.15$ (this is fully associated with the same parameter as $\left.c_{P}\right), c_{R}^{\beta}=0.1, c_{R}^{\mu}=0$.

the frequency of the bacteriocin profiles, such as the cost of toxin production and immunity (Figure 6), but also transmission rate $\beta$ and the effectiveness of invasion by the producer strain $k_{P}$ (see Supporting Figure 11).

\section{Discussion}

This paper was motivated by two main questions: firstly, the role of epidemiological processes in bacteriocin dynamics and diversity in S. pneumoniae and similar species; and secondly, whether these dynamics could generate differences in duration of carriage and therefore impact on the dynamics of antibiotic resistance.

We have shown that observed patterns of high bacteriocin diversity arise readily in epidemiological models. Due to uncertainty around how the inhibitory activity of bacteriocins translates to effects at the epidemiological scale, we have explored a range of model structures and parameter values. These models generate diversity robustly: coexistence of different bacteriocin profiles is found in all model structures for a wide range of parameters. On the other hand, the specifics of this coexistence (for example, which bacteriocin profiles coexist and at what frequencies) depend on both parametrisation and model structure. The sensitivity to bacteriocin characteristics such as the cost of toxin production may explain why the characteristics (e.g. prevalence and composition of bacteriocin loci) of known bacteriocin systems are so different.

In addition to the robust diversity in bacteriocin profiles in epidemiological models, we can also draw other general insights from these results.

Firstly, the inclusion of transmission dynamics allows different combinations of strains to coexist. Previous work on bacteriocin-specif within-host models suggests all three strains (producer, non-producer and immune) must be present to allow diversity to be maintained $[5,8]$. The epidemiological costs model also allows coexistence of the producer and non-producer strain without the immune strain. This difference arises because of differences in strain interactions at the within-host and epidemiological scale (Figure 1). At the within-host scale, the producer strain kills the non-producer strain: the producer strain benefits indirectly, through decreased overall bacterial density. At the epidemiological scale, the producer strain replaces the non-producer strain: the producer strain benefits directly from this interaction. Thus, at the epidemiological scale, the fitness of the producer strategy is more strongly dependent on the frequency of the non-producer. This gives rise to negative frequency-dependent selection, allowing stable coexistence of the producer and non-producer. 
Secondly, in S. pneumoniae, some bacteriocin systems, such as the pld locus [27] and the circular toxin pneumocyclicin [28], are found on only a subset of strains. Others, such as the blp $[23,24,17,25]$ and $c i b[26]$ loci, are ubiquitous. Such ubiquity is not consistent with insights from within-host models, or either of our basic models (head-to-head competition and epidemiological costs models): all stable equilibria require the presence of the non-producer strain, because toxin production is only beneficial when susceptible strains are present. Our results highlight two mechanisms which allow toxin-producer to exist in absence of non-producers (in other words, mechanisms that allow the bacteriocin locus to fully invade the bacterial population). Firstly, exclusion of the non-producer strain is seen in the hybrid model, when the toxins provide an advantage in both invasion of already colonised hosts and colonisation of uncolonised hosts (i.e. $\rho>0$ in Equation 3 - a biological interpretation would be that toxins are effective against both the focal and other species). Toxin production is maintained in absence of the non-producer strain because it provides a benefit despite the abscence of the non-producer. Secondly, in the pherotype model, immunity is not protective against toxins released by a different pherotype, strains of one pherotype are therefore susceptible to toxins from another pherotype. Toxin production is therefore beneficial even in absence of the non-producer strain. Indeed, the $c i b$ and $b l p$ loci are both associated with a signalling locus (i.e. pherotype), suggesting this may be the explanation for their ubiquity.

In the second part of the paper, we have shown that bacteriocins are a plausible candidate for involvement in resistance dynamics. Different model structures and parametrisations robustly predict differences in duration of carriage of different bacteriocin profiles, although for most parametrisations, these differences are relatively modest. Duration of carriage is predicted to modulate the fitness of antibiotic resistance [13]; we therefore expect an association between antibiotic resistance and bacteriocin profile and indeed observe this in a model incorporating both bacteriocin and resistance dynamics.

Testing this predicted association empirically is possible in theory, but would prove challenging given our current understanding of bacteriocin systems. Firstly, the direction of association between bacteriocin profile and antibiotic resistance depends on parametrisation. It is therefore unclear whether we expect antibiotic resistance to be associated with the producer, non-producer or immune phenotype. Secondly, the mapping between these modelled phenotypes and observed genotypes is non-trivial: although toxin and immunity genes can be identified, the effect on phenotype is less clear when multiple toxin and immunity genes are present on a genome (either because of the presence of a multi-toxin bacteriocin system or the presence of multiple different systems on one genome). Furthermore, in some bacteriocin systems, additional factors may affect the association between toxin and immunity genes; for example, a large proportion of $b l p$ systems may not be able to secrete pheromones and toxins because of an impaired transporter [29] and there is evidence of regulatory interplay between the $b l p$ and cib systems [30, 31]. Thus, although we have shown bacteriocins are a plausible candidate for involvement in resistance dynamics, more specific predictions about this involvement will require a more detailed understanding of specific bacteriocin systems.

In summary, we have shown that diversity in bacteriocin profiles arises robustly in epidemiological models of bacteriocin dynamics; that the nature of this diversity depends on the specifics of how bacteriocins impact epidemiological processes; and that diversity at bacteriocin loci is a plausible candidate for also maintaining diversity at resistance loci. This insights arise from a series of models that approach bacteriocins dynamics from a high level of abstraction, rather than representing a specific bacteriocin system. Generating more specific insights into particular bacteriocins systems will require models informed by the biology of the specific system. Therefore, a more complete understanding of the role of bacteriocins in bacterial ecology will need a more specific characterisation of their effects on transmission, invasion, within-host competition and duration of carriage.

\section{Acknowledgments}

We thank Marc Lipsitch for comments on the manuscript. This work was funded by the National Institutes of Health (MIDAS) (SL and CF grant ref: U01GM110721-01), the Li Ka Shing foundation (SL and CF); and a Sir Henry Dale Fellowship, jointly funded by Wellcome and the Royal Society (NJC, grant ref: 104169/Z/14/Z) 


\section{References}

[1] Hawlena H, Bashey F, Lively CM. Bacteriocin-mediated interactions within and between coexisting species. Ecology and Evolution. 2012;2(10):2521-2526.

[2] Riley MA, Wertz JE. Bacteriocins: evolution, ecology, and application. Annual Reviews in Microbiology. 2002;56(1):117-137.

[3] Miller EL, Abrudan MI, Roberts IS, Rozen DE. Diverse ecological strategies are encoded by Streptococcus pneumoniae bacteriocin-like peptides. Genome biology and evolution. 2016;8(4):1072-1090.

[4] Levin B. Frequency-dependent selection in bacterial populations. Philosophical Transactions of the Royal Society of London B, Biological Sciences. 1988;319(1196):459-472.

[5] Durrett R, Levin S. Allelopathy in spatially distributed populations. Journal of Theoretical Biology. 1997;185(2):165-171.

[6] Frean M, Abraham ER. Rock-scissors-paper and the survival of the weakest. Proceedings of the Royal Society of London Series B: Biological Sciences. 2001;268(1474):1323-1327.

[7] Czárán TL, Hoekstra RF, Pagie L. Chemical warfare between microbes promotes biodiversity. Proceedings of the National Academy of Sciences. 2002;99(2):786-790.

[8] Kerr B, Riley MA, Feldman MW, Bohannan BJ. Local dispersal promotes biodiversity in a real-life game of rock-paper-scissors. Nature. 2002;418(6894):171.

[9] Hjálmarsdóttir MÁ, Gumundsdóttir PF, Erlendsdóttir H, Kristinsson KG, Haraldsson G. Cocolonization of pneumococcal serotypes in healthy children attending day care centers: molecular versus conventional methods. The Pediatric infectious disease journal. 2016;35(5):477-480.

[10] Kamng'ona AW, Hinds J, Bar-Zeev N, Gould KA, Chaguza C, Msefula C, et al. High multiple carriage and emergence of Streptococcus pneumoniae vaccine serotype variants in Malawian children. BMC Infectious Diseases. 2015;15(1):234.

[11] Turner P, Hinds J, Turner C, Jankhot A, Gould K, Bentley SD, et al. Improved detection of nasopharyngeal cocolonization by multiple pneumococcal serotypes by use of latex agglutination or molecular serotyping by microarray. Journal of Clinical Microbiology. 2011;49(5):1784-1789

[12] Kelsic ED, Zhao J, Vetsigian K, Kishony R. Counteraction of antibiotic production and degradation stabilizes microbial communities. Nature. 2015;521(7553):516.

[13] Lehtinen S, Blanquart F, Croucher NJ, Turner P, Lipsitch M, Fraser C. Evolution of antibiotic resistance is linked to any genetic mechanism affecting bacterial duration of carriage. Proceedings of the National Academy of Sciences of the United States of America. 2017 jan;114(5):1075-1080.

[14] Lehtinen S, Blanquart F, Lipsitch M, Fraser C, with the Maela Pneumococcal Collaboration, et al. On the evolutionary ecology of multidrug resistance in bacteria. PLoS Pathogens. 2019;15(5):e1007763.

[15] Colijn C, Cohen T, Fraser C, Hanage W, Goldstein E, Givon-Lavi N, et al. What is the mechanism for persistent coexistence of drug-susceptible and drug-resistant strains of Streptococcus pneumoniae? Journal of The Royal Society Interface. 2010;7(47).

[16] Lees JA, Croucher NJ, Goldblatt D, Nosten F, Parkhill J, Turner C, et al. Genome-wide identification of lineage and locus specific variation associated with pneumococcal carriage duration. bioRxiv. 2017;p. 107086.

[17] Dawid S, Roche AM, Weiser JN. The blp bacteriocins of Streptococcus pneumoniae mediate intraspecies competition both in vitro and in vivo. Infection and Immunity. 2007;75(1):443451. 
[18] Valente C, Dawid S, Pinto FR, Hinds J, Simões AS, Gould KA, et al. The blp locus of Streptococcus pneumoniae plays a limited role in the selection of strains that can cocolonize the human nasopharynx. Appl Environ Microbiol. 2016;82(17):5206-5215.

[19] Inc WR. Mathematica, Version 11.2;. Champaign, IL, 2017.

[20] Corander J, Fraser C, Gutmann MU, Arnold B, Hanage WP, Bentley SD, et al. Frequencydependent selection in vaccine-associated pneumococcal population dynamics. Nature Ecology \& Evolution. 2017;1(12):1950.

[21] Colijn C, Corander J, Croucher N. Designing ecologically-optimised vaccines using population genomics. bioRxiv. 2019;p. 672733.

[22] Azarian T, Martinez PP, Arnold BJ, Grant LR, Corander J, Fraser C, et al. Predicting evolution using frequency-dependent selection in bacterial populations. bioRxiv. 2019;p. 420315 .

[23] de Saizieu A, Gardès C, Flint N, Wagner C, Kamber M, Mitchell TJ, et al. Microarray-based identification of a novelStreptococcus pneumoniae regulon controlled by an autoinduced peptide. Journal of Bacteriology. 2000;182(17):4696-4703.

[24] Reichmann P, Hakenbeck R. Allelic variation in a peptide-inducible two-component system of Streptococcus pneumoniae. FEMS microbiology letters. 2000;190(2):231-236.

[25] Lux T, Nuhn M, Hakenbeck R, Reichmann P. Diversity of Bacteriocins and Activity Spectrum in Streptococcus pneumoniae. Journal of Bacteriology. 2007 Nov;189(21):77417751.

[26] Guiral S, Mitchell TJ, Martin B, Claverys JP. Competence-programmed predation of noncompetent cells in the human pathogen Streptococcus pneumoniae: genetic requirements. Proceedings of the National Academy of Sciences of the United States of America. 2005;102(24):8710-8715.

[27] Maricic N, Anderson ES, Opipari AE, Emily AY, Dawid S. Characterization of a Multipeptide Lantibiotic Locus in Streptococcus pneumoniae. mBio. 2016;7(1):e01656-15.

[28] Bogaardt C, van Tonder AJ, Brueggemann AB. Genomic analyses of pneumococci reveal a wide diversity of bacteriocins-including pneumocyclicin, a novel circular bacteriocin. BMC Genomics. 2015;16(1):554.

[29] Son MR, Shchepetov M, Adrian PV, Madhi SA, de Gouveia L, von Gottberg A, et al. Conserved mutations in the pneumococcal bacteriocin transporter gene, blpA, result in a complex population consisting of producers and cheaters. MBio. 2011;2(5):e00179-11.

[30] Wholey WY, Kochan TJ, Storck DN, Dawid S. Coordinated bacteriocin expression and competence in Streptococcus pneumoniae contributes to genetic adaptation through neighbor predation. PLoS pathogens. 2016;12(2):e1005413.

[31] Kjos M, Miller E, Slager J, Lake FB, Gericke O, Roberts IS, et al. Expression of Streptococcus pneumoniae bacteriocins is induced by antibiotics via regulatory interplay with the competence system. PLoS pathogens. 2016;12(2):e1005422. 


\section{Supporting Information}

\section{Models and analysis}

\subsection{Head-to-head competition model}

The direct competition model (main text Equation 1) admits eight equilibrium solutions. We assess the stability of these equilibria using linear stability analysis: i.e. classifying their stability based on the sign of the real part of the eigenvalues of the system's Jacobian matrix. All apart from one of these equilibria are unstable (i.e. the real part of at least one of the eigenvalues of the Jacobian is positive) given the constraints on parameter values in Table 1 (main text). This stable equilibrium is:

$$
I_{P}=\frac{k_{N}(\beta-\mu)}{\beta\left(k_{P}+k_{M}+k_{N}\right)}, I_{M}=\frac{k_{P}(\beta-\mu)}{\beta\left(k_{P}+k_{M}+k_{N}\right)}, I_{N}=\frac{k_{M}(\beta-\mu)}{\beta\left(k_{P}+k_{M}+k_{N}\right)}
$$

The eigenvalues of this equilibrium are:

$$
\left(\mu-\beta,-\beta \sqrt{-\frac{k_{P} k_{M} k_{N}(\beta-\mu)^{2}}{\beta^{2}\left(k_{P}+k_{M}+k_{N}\right)}}, \beta \sqrt{-\frac{k_{P} k_{M} k_{N}(\beta-\mu)^{2}}{\beta^{2}\left(k_{P}+k_{M}+k_{N}\right)}}\right)
$$

In the parameter space of interest (Table 1 main text), the first eigenvalue is always real and negative and the last two eigenvalues are always purely imaginary and of opposite signs. This system therefore gives rise to stable periodic trajectories (i.e. oscillations) around the equilibrium point (SI Figure 1).
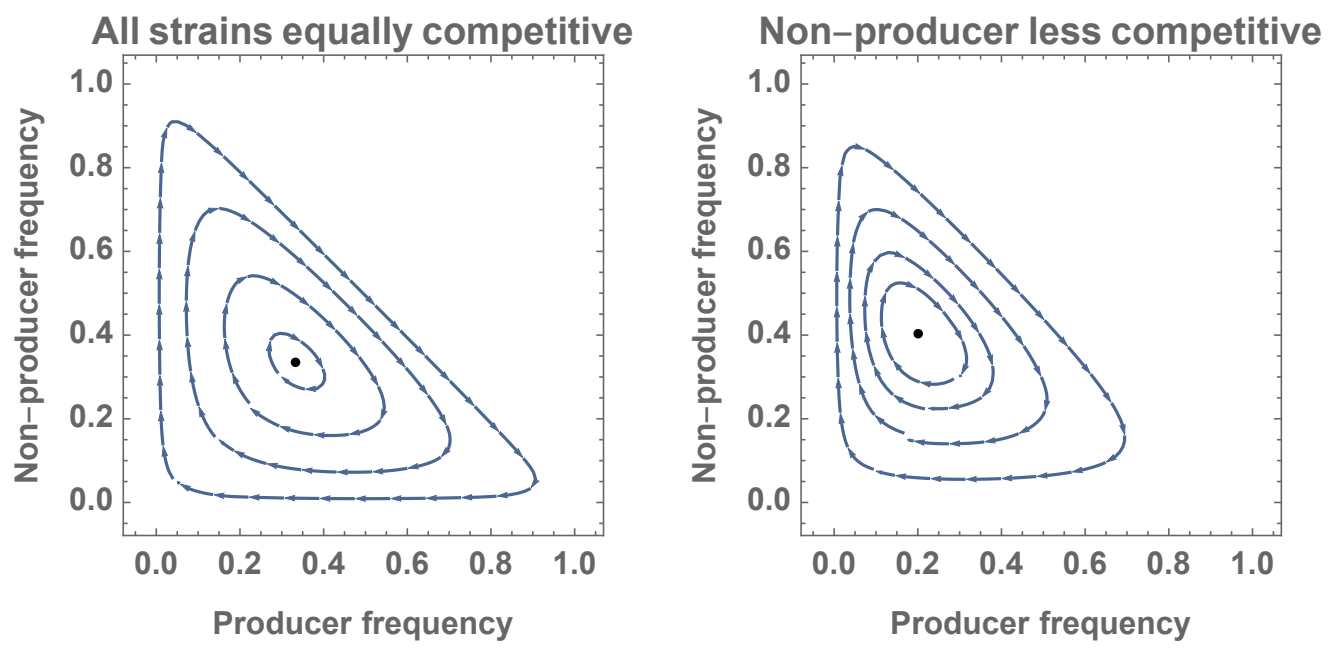

Figure 1: Trajectories showing stable oscillation of producer, immune and non-producer strain frequencies in the direct competition model. The different trajectories arise from a different set of initial conditions. Frequencies of the three strains always add up to 1, the frequency of the immune strain is therefore one minus the sum of the other two. Dots show the equilibrium value around which the trajectories oscillate. Parameter values are $\beta=2, \mu=1, k_{P}=0.5, k_{M}=$ $0.5, k_{N}=0.5$ (left plot) and $k_{N}=0.25$ (right plot). 


\subsection{Epidemiological costs model}

The epidemiological costs model (main text Equation 2) has seven solutions. It was not possible to derive analytical bounds for the stability of these solutions. The results presented in the main text for this model are therefore derived by numerically computing the eigenvalues of the equilibria at various parameter values. Note that when costs affect the transmission rate only $\left(c_{P}^{\mu}=c_{M}^{\mu}=0\right)$ we can analytically exclude stability of the the producer and immune strain without the sensitive strain (never stable in the parameter space of interest) and the immune strain alone (only stable when $c_{P}^{\beta}=2 c_{M}^{\beta}+\frac{\beta}{\mu}\left(1-c_{M}^{\beta}\right)^{2}$ ).

\subsection{Generalised version of basic models}

The two models presented in the main text represent specific parametrisations of a more general model of bacteriocin dynamics. In the models in the main text:

- the fitness cost of toxins and immunity is assumed to affect either the rate of invasion (headto-head competition model) or the transmission and/or clearance rate (epidemiological costs model).

- invasion is assumed only to occur for specific strain pairs, and only in one direction.

Relaxing these assumptions gives a more general formulation of the model, in which $k_{A B}$ is the rate at which strain $\mathrm{A}$ invades strain $\mathrm{B}$. The dynamics of this model are described by:

$$
\begin{array}{r}
\left.\frac{d I_{P}}{d t}=\beta_{P} I_{P} X+\left(\beta_{P} k_{P M}-\beta_{M} k_{M P}\right) I_{M} I_{P}\right)+\left(\beta_{P} k_{P N}-\beta_{N} k_{N P}\right) I_{N} I_{P}-\mu_{P} I_{P} \\
\frac{d I_{M}}{d t}=\beta_{M} I_{M} X+\left(\beta_{M} k_{M N}-\beta_{N} k_{N M}\right) I_{N} I_{M}+\left(\beta_{M} k_{M P}-\beta_{P} k_{P M}\right) I_{P} I_{M}-\mu_{M} I_{M} \\
\frac{d I_{N}}{d t}=\beta_{N} I_{N} X+\left(\beta_{N} k_{N P}-\beta_{P} k_{P N}\right) I_{P} I_{N}+\left(\beta_{N} k_{N M}-\beta_{M} k_{M N}\right) I_{M} I_{N}-\mu_{N} I_{N}
\end{array}
$$

Setting $\beta_{P}=\beta_{M}=\beta_{N}, \mu_{P}=\mu_{M}=\mu_{N}$ and $k_{N P}=k_{P M}=k_{M N}=0$ recovers the head to head competition model. Setting $k_{N P}=k_{P M}=k_{M P}=k_{M N}=k_{N M}=0$ recovers the epidemiological costs model.

This more general formulation allows us to examine the impact of assuming bacteriocins are defensive (i.e. prevent invasion already colonised hosts) rather than offensive (i.e. allow invasion). In the main text, we describe bacteriocins as offensive (i.e. enabling invasion of ). It could also be that the role of bacteriocins is defensive or a combination of both.

When the transmission rates do not differ between strains $\left(\beta_{M}=\beta_{N}=\beta_{P}\right)$, (i.e. the head to head model competition model and the epidemiological costs model when costs impact clearance rate only), the distinction between offensive and defensive has no impact on epidemiological dynamics. This is because, when transmission rates are equal, the interaction between strains $A$ and $B$ in the dynamics of strain $A$ simplifies to $\beta\left(k_{A B}-k_{B A}\right) I_{A} I_{B}$. Model dynamics therefore depend on the relative rates of invasion. Under these circumstances, describing bacteriocins as offensive (the producer strain invades the non-producer at rate $k$, all other invasion parameters are zero) is mathematically equivalent to describing bacteriocins as defensive (all strains invade each other at rate $k$, except the non-producer strain, which cannot invade the producer strain).

The equivalence between offensive and defensive does not hold when transmission rates are equal (i.e. epidemiological costs model when costs also affect transmission rate). This is because the rate of successful invasion by strain $A$ depends on $\beta_{A}$, whereas the rate of strain $A$ being invaded by strain $B$ depends on $\beta_{B}$. As a result, the interaction between strains $A$ and $B$ in the dynamics of strain $A\left(\left(\beta_{A} k_{A B}-\beta_{B} k_{B A}\right) I_{A} I_{B}\right.$ does not simplify.

However, we find that in practice, this does not have an impact on which strains are observed to coexist. We set $k_{P N}=k_{P M}=k_{M P}=k_{M N}=k_{N M}=k$ and $k_{N P}=0$ in the general model, giving rise to an epidemiological costs model with defensive bacteriocins. We find that the parameter ranges in which different bacteriocin profiles coexist are identical to the epidemiological costs model in the main text in which bacteriocins are offensive (SI Figure 2). 

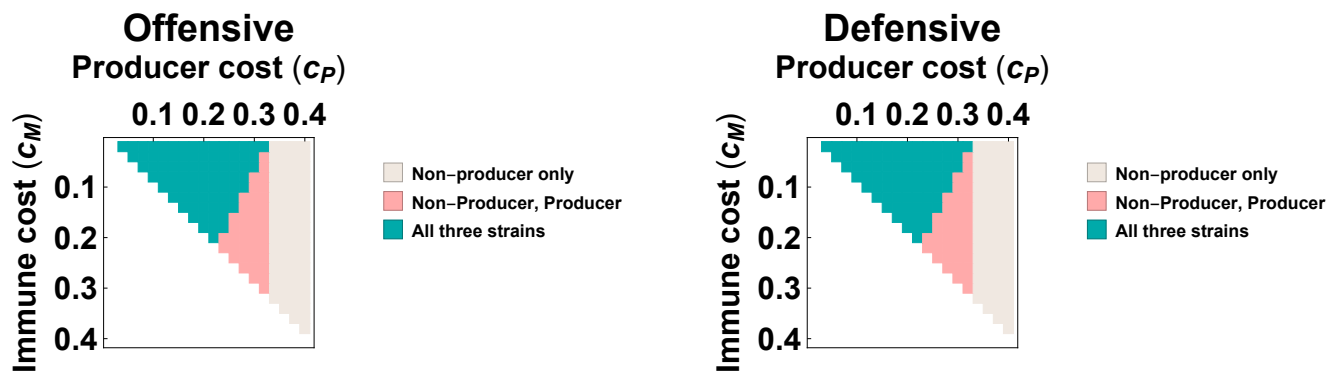

Figure 2: Strains present at equilibrium in the epidemiological costs model (with costs affecting transmission) when the action of bacteriocins is offensive $k_{N P}=k_{P M}=k_{M P}=k_{M N}=k_{N M}=$ 0 and $k_{P N}=0.5$, corresponding to main text Figure 4) and defensive $\left(k_{P N}=k_{P M}=k_{M P}=\right.$ $k_{M N}=k_{N M}=0.5$ and $\left.k_{N P}=0\right)$. Other parameters are $\beta=2$ and $\mu=1$.

\subsection{Pherotype models}

There are two things to note about how the two pherotype models in the main text have been written. Firstly, whether a producer strain is able to invade a host colonised with a producer strain of the other pherotype would dependent on assumptions about the timing of toxin and immunity expression. However, in line with the point made in Supporting Information Section 1.1, this does not impact model dynamics: the rate at which the pherotype A producer replaces the pherotype $\mathrm{B}$ producer $\left(\beta_{P} k_{P} I_{a, P} I_{b, P}\right)$ is equal to the rate at which the pherotype $\mathrm{B}$ producer replaces the pherotype A producer. These terms therefore cancel out and we have not included them in main text Equations 4 and 5.

Secondly, the non-producer strain is always susceptible to the toxin - producer strains can therefore replace non-producer strains regardless of pherotype. Non-producers of the two pherotypes are therefore ecologically indistinguishable and modelling the two non-producers strains as a single strain would give identical results.

For both the direct competition and epidemiological cost models, the versions including pherotype give rise to a large number of solutions. Similarly to the epidemiological costs model, it was not possible to determine analytical bounds for the stability of these solutions, the results in the main text are therefore derived by computing the eigenvalues evaluated at the equilibrium points numerically for various parameter ranges.

\subsection{Resistance model}

\subsubsection{Model structure}

We model a species that is carried asymptomatically most of the time (e.g. S. pneumoniae) - the antibiotic exposure of hosts is therefore independent of whether they are colonised and therefore equal to average consumption rate of the antibiotic $(\tau)$. Interactions between the three bacteriocin profiles are the same as in the basic epidemiological costs model (Equation 2). In addition, each profile can be either antibiotic sensitive $(S)$ or antibiotic resistant $(R)$, giving rise to six possible strains $(P S, P R, M S, M R, N S, N R)$. Antibiotic sensitive strains are subject to an additional clearance rate $\tau$ (we assume immediate clearance in response to antibiotics). Resistance carries a fitness cost, which, for clarity, we assume affects transmission $\left(c_{R}^{\beta}\right)$ in the main text, such that $\beta_{P R}=\left(1-c_{R}^{\beta}\right) \beta_{P S}=\left(1-c_{R}^{\beta}\right)\left(1-c_{P}^{\beta}\right) \beta$ and similarly for strains $M S$ and $N S\left(\right.$ where $\left.\beta_{N S}=\beta\right)$.

The dynamics of this system are described by: 


$$
\begin{array}{r}
\frac{d I_{P S}}{d t}=\beta_{P S} I_{P S}\left(X+k_{P}\left(I_{N S}+I_{N R}\right)\right)-(\mu+\tau) I_{P S} \\
\frac{d I_{M S}}{d t}=\beta_{M S} I_{M S} X-(\mu+\tau) I_{M S} \\
\frac{d I_{N S}}{d t}=\beta_{N S} I_{N S} X-k_{P}\left(\beta_{P R} I_{P R}+\beta_{P S} I_{P S}\right) I_{N S}-(\mu+\tau) I_{N S} \\
\frac{d I_{P R}}{d t}=\beta_{P R} I_{P R}\left(X+k_{P}\left(I_{N S}+I_{N R}\right)\right)-\mu I_{P R} \\
\frac{d I_{M R}}{d t}=\beta_{M R} I_{M R} X-\mu I_{M R} \\
\frac{d I_{N R}}{d t}=\beta_{N R} I_{N R} X-k_{P}\left(\beta_{P R} I_{P R}+\beta_{P S} I_{P S}\right) I_{N R}-\mu I_{N R}
\end{array}
$$

\subsubsection{Results}

The resistance model was not analytically tractable. All results are therefore derived by numerically simulating the system. Results presented in main text Figure 6 are for $\mathrm{t}=100000$ (at which point the system appears to have reached equilibrium (SI Figure 3), with initial conditions $I_{P R}=I_{P S}=I_{M R}=I_{M S}=I_{N R}=I_{N S}=\frac{1}{6}$.

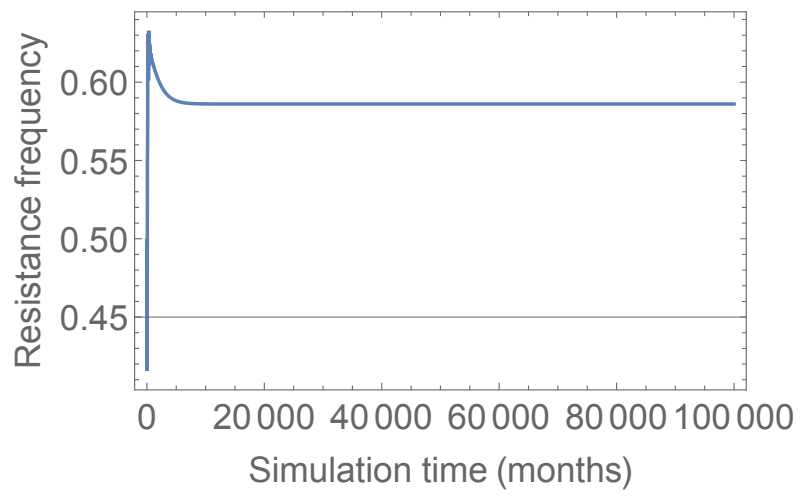

Figure 3: Total resistance frequency as a function of time in a numerical simulation of the resistance model with parameters $\beta=4, \mu=1, k_{P}=0.5, c_{p}^{\beta}=0.25, c_{M}^{\beta}=0.15, c_{R}^{\beta}=0.1$, and all other costs 0 (similar to the main text Figure 5)

\section{Additional results relating to resistance dynamics}

\subsection{Head-to-head competition model}

In the direct competition model, at equilibrium the mean duration of carriage of each strain is given by:

$$
\begin{aligned}
D_{P} & =\frac{1}{\mu+k_{M} \beta I_{M} *} \\
D_{M} & =\frac{1}{\mu+k_{N} \beta I_{N} *} \\
D_{N} & =\frac{1}{\mu+k_{P} \beta I_{P} *}
\end{aligned}
$$

The $I *$ variables indicate the equilibrium value (i.e. the value around which the strain frequencies oscillate - SI Figure 1). It is these durations which matter for resistance dynamics rather than the duration of carriage at any particular point during oscillation (which will depend on the magnitude of the oscillation). 

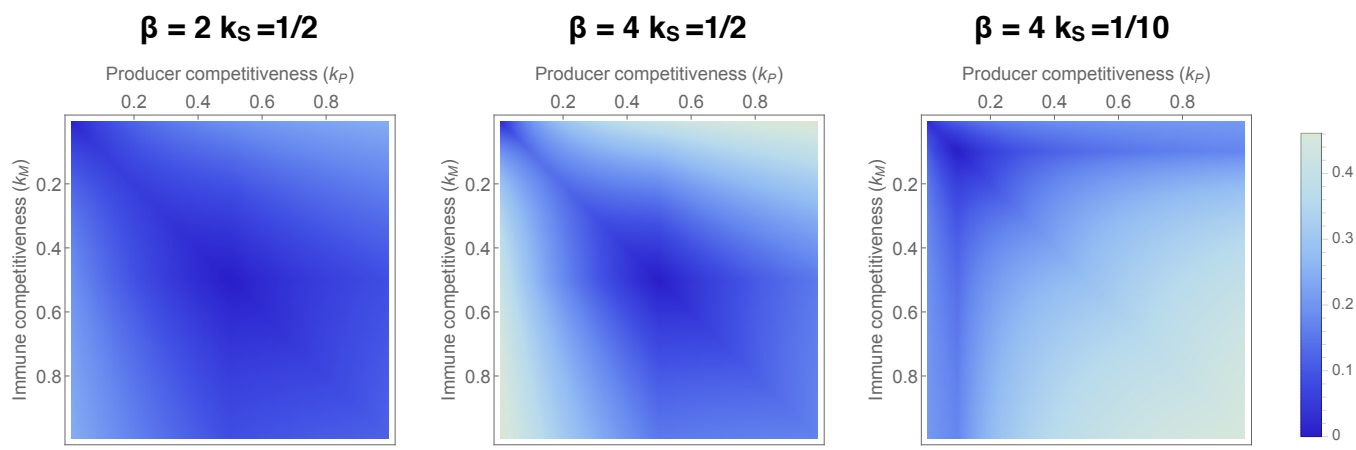

Figure 4: Variation in duration of carriage of bacteriocin profiles in the direct competition model. The range of duration of carriage of bacteriocin profiles (i.e. longest duration of carriage minus shortest duration of carriage), with $\mu=1$.

SI Figure 4 shows how these differences in duration depend on parametrisation - the largest differences are observed when transmission rate is high and when differences in the $k$ parameters are the greatest. However, as with the epidemiological costs model, these differences are relatively modest. Therefore, in a model explicitly incorporating resistance dynamics (Equations 4, with $\tau$ being the population antibiotic consumption and $c_{R}$ being the cost of resistance, which we model as associated with transmission), the range of antibiotic consumption rates at which coexistence arises is also relatively narrow (SI Figure 5).

$$
\begin{array}{r}
\frac{d I_{P S}}{d t}=\beta I_{P S}\left(X+k_{P}\left(I_{N S}+I_{N R}\right)\right)-k_{M} \beta\left(I_{M S}+I_{M R}\right) I_{P S}-(\mu+\tau) I_{P S} \\
\frac{d I_{M S}}{d t}=\beta I_{M S}\left(X+k_{M}\left(I_{P S}+I_{P R}\right)\right)-k_{N} \beta\left(I_{N S}+I_{N R}\right) I_{M S}-(\mu+\tau) I_{M S} \\
\frac{d I_{N S}}{d t}=\beta I_{N S}\left(X+k_{N}\left(I_{M S}+I_{M R}\right)\right)-k_{P} \beta\left(I_{P S}+I_{P R}\right) I_{N S}-(\mu+\tau) I_{N S} \\
\frac{d I_{P R}}{d t}=\left(1-c_{R}\right) \beta I_{P R}\left(X+k_{P}\left(I_{N S}+I_{N R}\right)\right)-k_{M} \beta\left(I_{M S}+I_{M R}\right) I_{P R}-\mu I_{P R} \\
\frac{d I_{M R}}{d t}=\left(1-c_{R}\right) \beta I_{M R}\left(X+k_{M}\left(I_{P S}+I_{P R}\right)\right)-k_{N} \beta\left(I_{N S}+I_{N R}\right) I_{M R}-\mu I_{M R} \\
\frac{d I_{N R}}{d t}=\left(1-c_{R}\right) \beta I_{N R}\left(X+k_{N}\left(I_{M S}+I_{M R}\right)\right)-k_{P} \beta\left(I_{P S}+I_{P R}\right) I_{N R}-\mu I_{N R}
\end{array}
$$

\subsection{The cost of resistance in clearance}

There is an important caveat related to the modelling of the cost of antibiotic resistance when it is associated with clearance rate. Whether the cost of resistance is modelled in transmission or in clearance will not affect the antibiotic consumption rate at which resistance is selected for as long as the cost affects all components of clearance.

To illustrate this, we consider a strain that can be cleared through two processes which occur at rates $\alpha$ and $\gamma$ and follow the reasoning in Lehtinen et al. [1]. If the cost of resistance affects transmission rate, the $R_{0}$ of the resistant strain is $\frac{\left(1-c_{R}\right) \beta}{\alpha+\gamma}$. The $R_{0}$ of the antibiotic sensitive strain is $\frac{\beta}{\alpha+\gamma+\tau}$, where $\tau$ is the population antibiotic consumption. The strain with the higher $R_{0}$ will out-compete the other - resistance is therefore selected for when $\tau>\frac{(\mu+\gamma) c_{R}}{1-c_{R}}$. If the cost is modelled in clearance and affects both components of clearance rate ( $\alpha$ and $\gamma)$, the expression for the $R_{0}$ of the resistant strain is identical and the result is the same.

However, if only one of the processes (say the one occurring at rate $\alpha$ ) is affected by the cost of clearance, the $R_{0}$ of the resistant strain is $\frac{\beta}{\left(\frac{\alpha}{\left(1-c_{R}\right)}+\gamma\right.}$. Resistance is therefore selected for when $\tau>\frac{\mu c_{R}}{1-c_{R}}$. If the cost of resistance affects clearance rate only, it is only the components of clearance rate affected by the cost of resistance that play a role in resistance dynamics.

In SI Figure 6, we examine how this affects the impact of bacteriocins on resistance dynamics. When the cost of antibiotic resistance affects both components of clearance (i.e. $\mu$ and the rate 


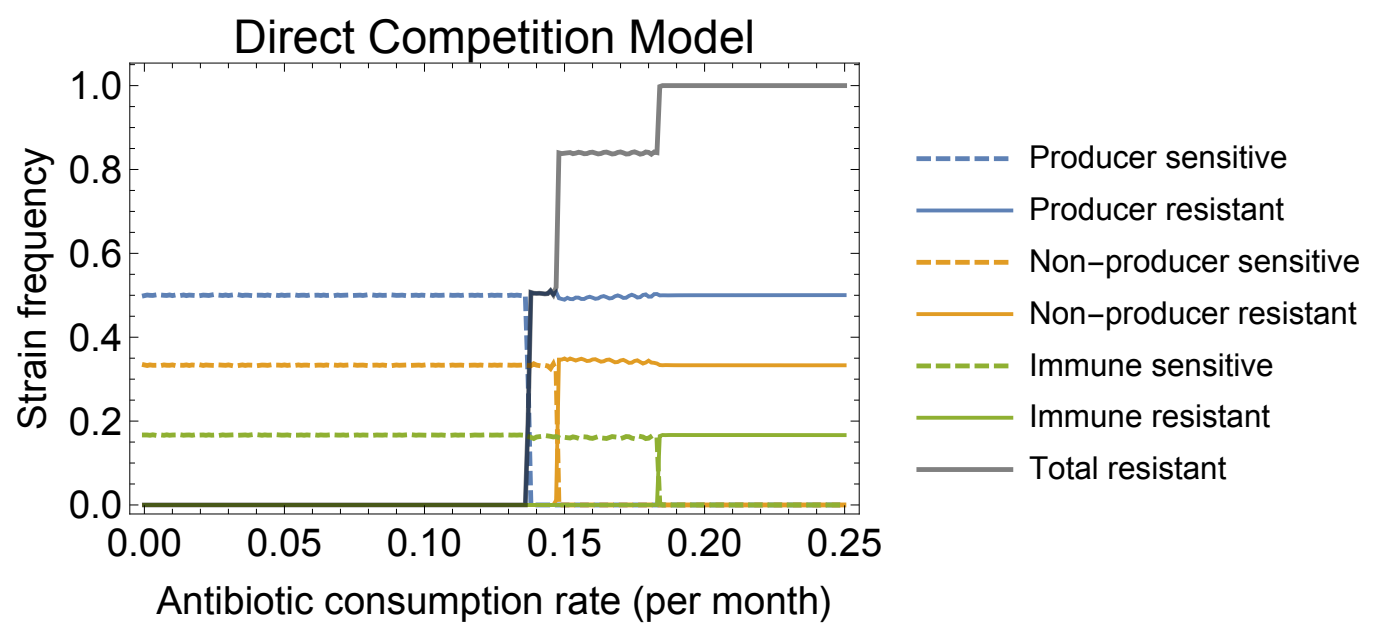

Figure 5: An example of the frequency of antibiotic resistance as a function of antibiotic consumption in the direct competition model. The parameter values are $\beta=4, \mu=1, c_{R}=0.1$, $k_{N}=0.75, k_{M}=0.5$ and $k_{P}=0.25$. The model was initialised so that the proportions of the different bacteriocin profiles were at equilibrium, which is why no oscillations are observed (i.e. at $t=0, I_{P S}+I_{P R}=I_{P}$ and $I_{P S}=I_{P R}$ - see Supporting Information Section 1.1 for values).

at which invasion of the resistant strain occurs), it does not matter whether the cost of antibiotic resistance is modelled in transmission or clearance - results are identical. However, if the cost of resistance is modelled as affecting $\mu$ only, the model's behaviour is sensitive to whether the cost of resistance is modelled in transmission or clearance. When the cost of resistance is modelled in clearance and bacteriocin costs are modelled in transmission, the model predicts no coexistence: all bacteriocin profiles have the same $\mu$ and therefore resistance becomes beneficial at the same antibiotic consumption for all strains. When the cost of resistance and bacteriocin costs are modelled in clearance, the bacteriocin costs introduce differences between $\mu_{P}, \mu_{M}$ and $\mu_{N}$, leading to a region of coexistence. 

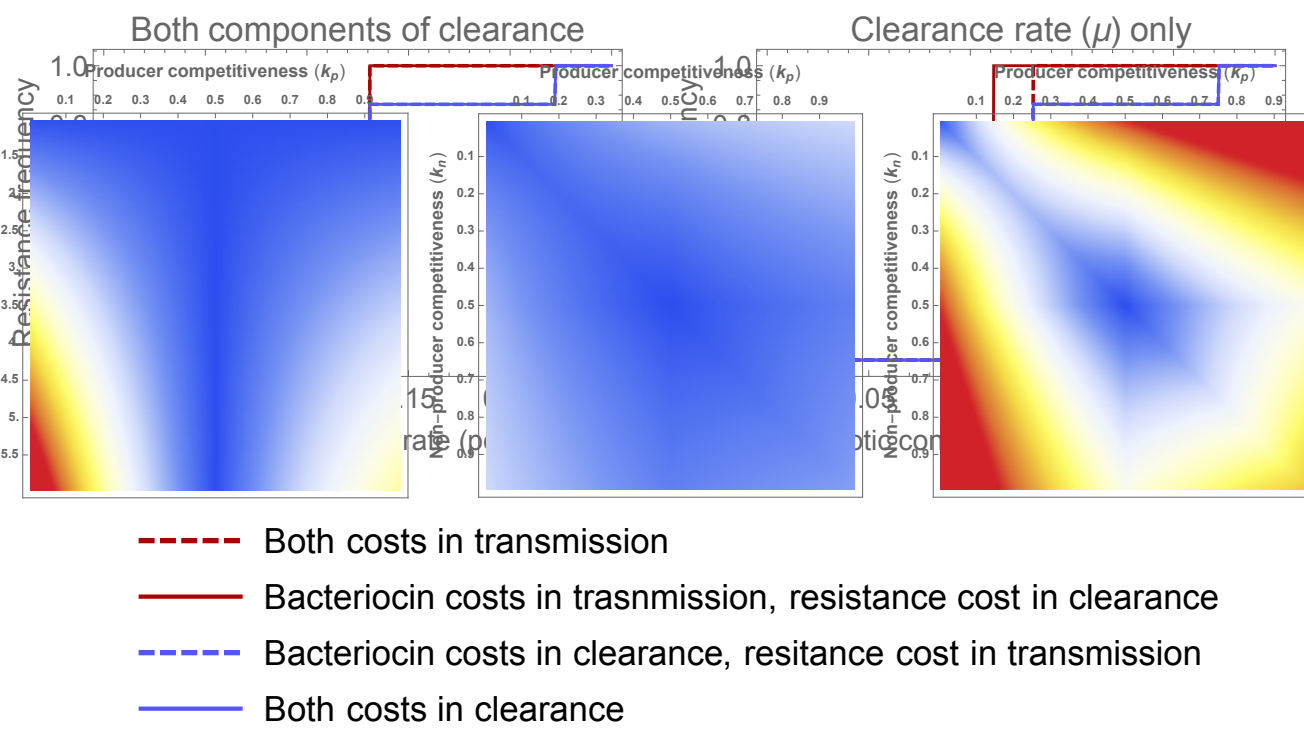

Figure 6: The effect of how the cost of antibiotic consumption is modelled in the epidemiological costs model. The cost can either affect both components of clearance (clearance rate $\mu$ and replacement of by the producer strain) (left) or just the clearance rate $\mu$ (right). The plots show equilibrium resistance frequencies as a function of antibiotic consumption rate for different ways of modelling costs: bacteriocins costs in transmission vs clearance (red vs blue) and resistance cost in transmission vs clearance (dashed vs solid).

\section{Additional Figures}
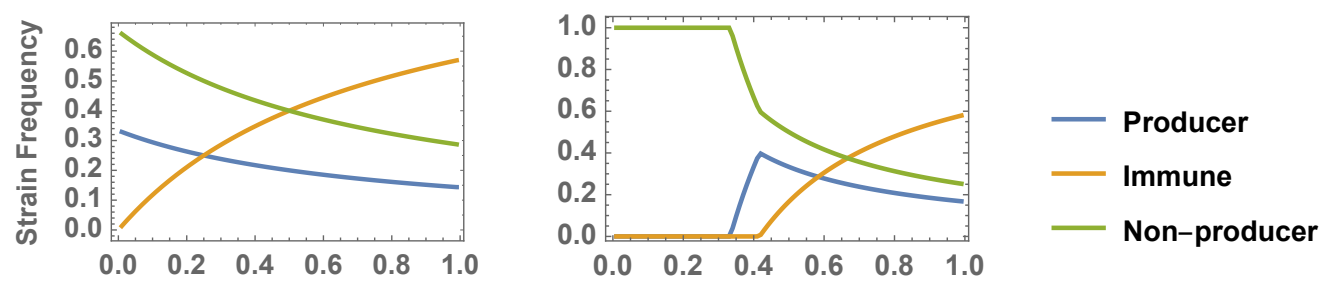

Producer competitiveness $\left(k_{P}\right)$

Figure 7: "Survival of the weakest" behaviour in the direct competition (left) and epidemiological costs model (right). The plots show strain frequencies at equilibrium as a function of the competitiveness of the producer strain $\left(k_{P}\right)$. The direct competition model always exhibits survival of the weakest: the frequency of the producer strain declines with its competitiveness. The epidemiological costs model only exhibits this behaviour when all three strains are present: in the two strain equilibrium, the frequency of the producer strain increases with its competitiveness. Other parameter values are $\beta=2, \mu=1$ for both models and $k_{N}=0.25, k_{M}=0.5$ for the direct competition model and $c_{P}=0.25, c_{M}=0.1$ for the epidemiological costs model. 


\section{Producer competitiveness $\left(k_{P}\right)$}

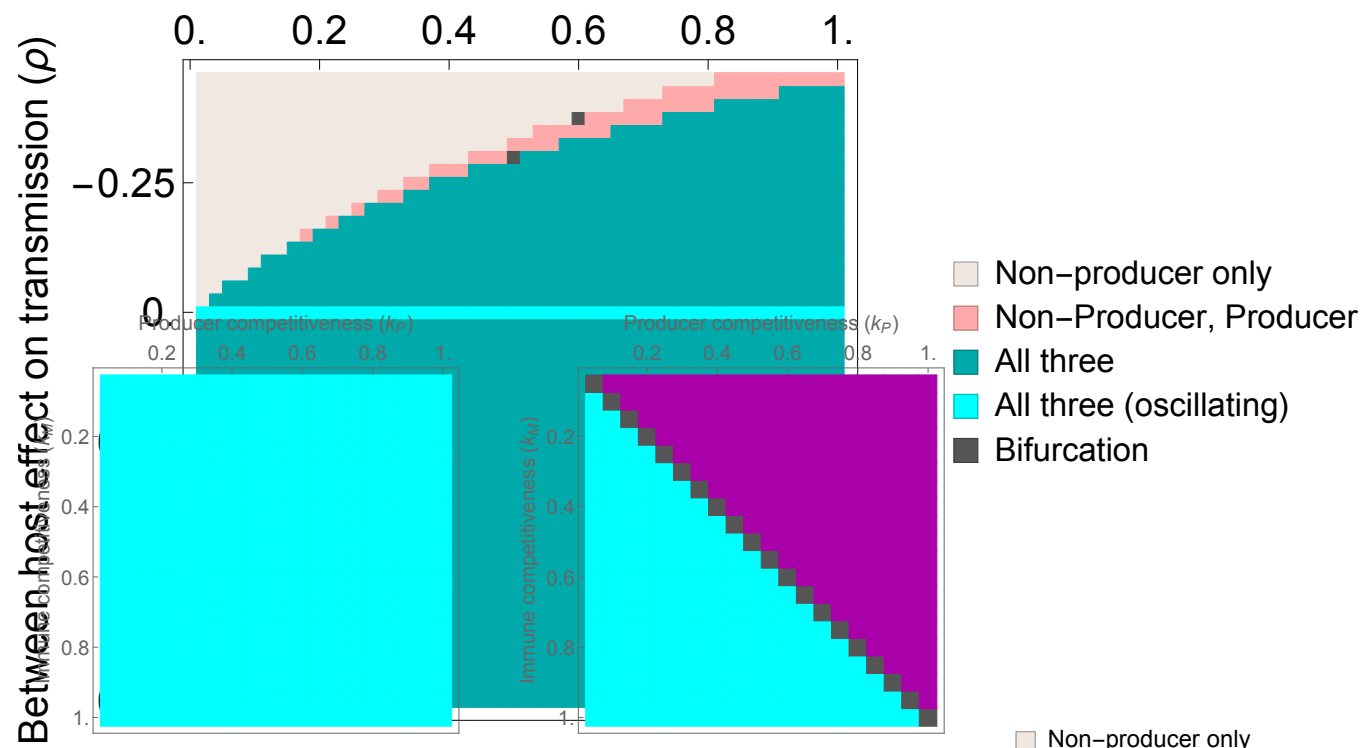

Figure 8: Introducing the epidemiological fitness difference in clearance rather than transmission in the hybrid model. The colours indicate which strains are present at equilibrium. Results are derived from a linear stability analysis and results shown are for asymptotically stable equilibria unless otherwise indicated. Model parameters were $\beta=2, \mu=1, k_{N}=0.5, k_{M}=0.5$.
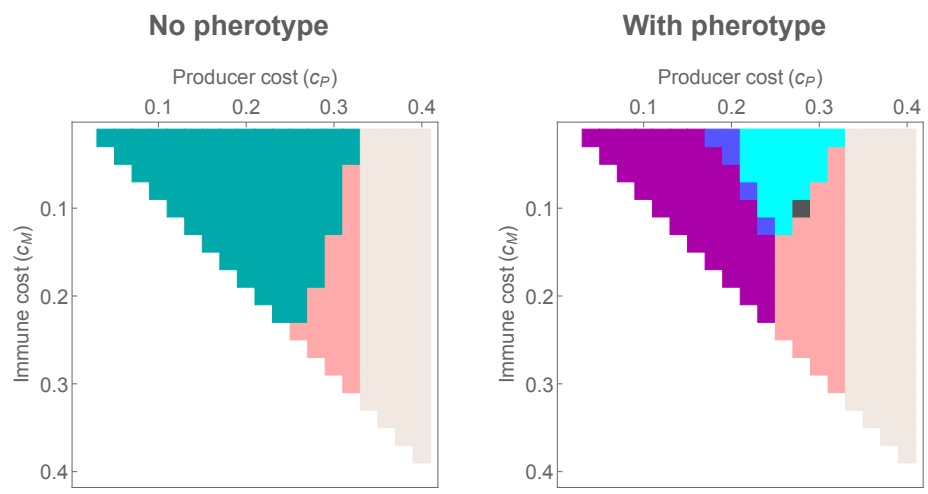

$\square$ Non-producer only

$\square$ Non-producer, Producer

- Producer only

Producer, Immune (oscillating)

All three

$\square$ All three (oscillating)

- Bifurcation

Figure 9: Effect of modelling the costs of toxin production and immunity in clearance in the epidemiological costs model. Parameters are identical to those for main text Figure 4, but the cost of bacteriocin and toxin production affects clearance rather than transmission rate. 

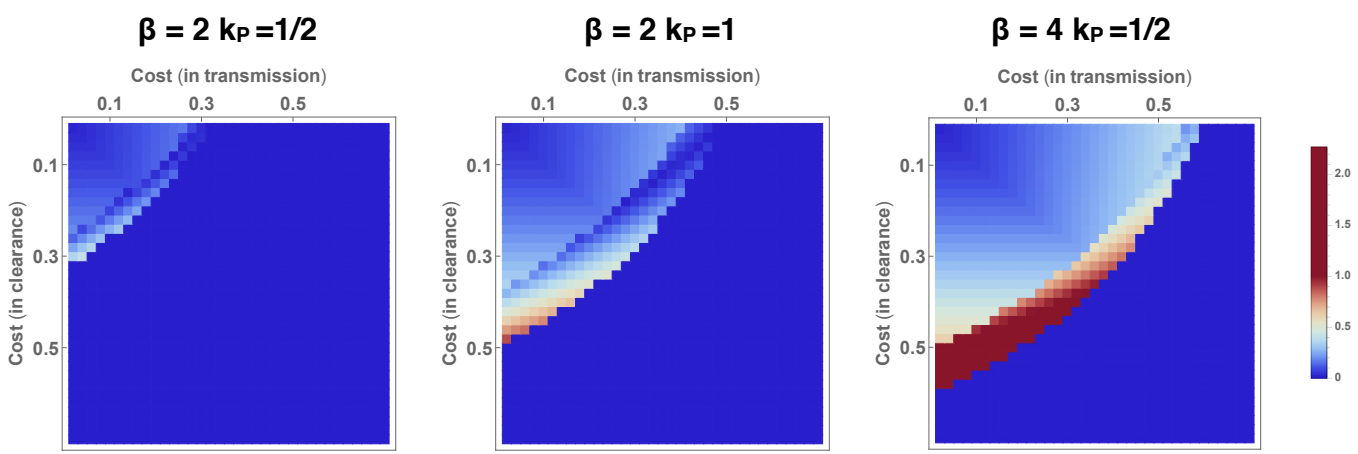

Figure 10: Variation in duration of carriage of bacteriocin profiles in the epidemiological costs model. The range of duration of carriage of bacteriocin profiles (i.e. longest duration of carriage minus shortest duration of carriage). The cost the on the $x$ and $y$ axes indicate the cost to the producer strain $\left(c_{P}^{\beta}\right.$ and $c_{P}^{\mu}$ respectively). The cost to the immune strain is half of the cost to the producer strain $c_{P}^{\beta}=2 c_{M}^{\beta}$ and $c_{P}^{\mu}=2 c_{M}^{\mu} \cdot \mu=1$. The area of solid blue (range of zero) arises because there is no coexistence of different bacteriocin profiles in this parameter space: the non-producer excludes the other two strains.

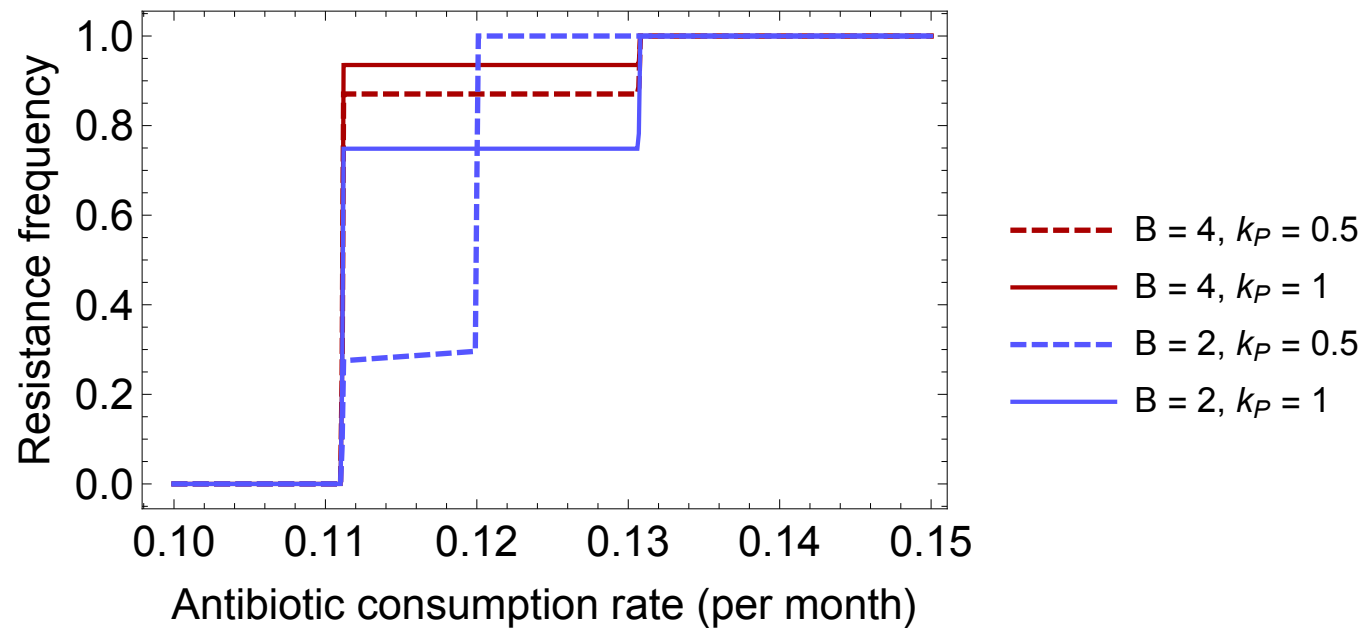

Figure 11: An example of how predicted resistance frequencies depend on transmission rate (red vs blue) and the effectiveness of invasion by the producer (dashed vs solid) in the epidemiological costs model. Other parameters are $\mu=1, c_{P}^{\beta}=0.25, c_{M}^{\beta}=0.15, c_{R}^{\beta}=0.1$ and all other costs zero. 


\section{References}

[1] Lehtinen S, Blanquart F, Croucher NJ, Turner P, Lipsitch M, Fraser C. Evolution of antibiotic resistance is linked to any genetic mechanism affecting bacterial duration of carriage. Proceedings of the National Academy of Sciences of the United States of America. 2017 jan;114(5):1075-1080. 